Revue Française de Civilisation Britannique

\title{
«On ne peut pas empêcher les concepts de voyager » : un entretien avec Christine Delphy
}

"You Can't Stop Concepts from Travelling": an Interview with Christine Delphy

\section{Marc Calvini-Lefebvre}

\section{(2) OpenEdition}

\section{Journals}

Édition électronique

URL : http://journals.openedition.org/rfcb/1817

DOI : $10.4000 /$ rfcb. 1817

ISSN : 2429-4373

Éditeur

CRECIB - Centre de recherche et d'études en civilisation britannique

Référence électronique

Marc Calvini-Lefebvre, « «On ne peut pas empêcher les concepts de voyager » : un entretien avec Christine Delphy », Revue Française de Civilisation Britannique [En ligne], XXIII-1 | 2018, mis en ligne le 20 mars 2018, consulté le 21 août 2020. URL : http://journals.openedition.org/rfcb/1817 ; DOI : https:// doi.org/10.4000/rfcb. 1817

Ce document a été généré automatiquement le 21 août 2020.

Revue française de civilisation britannique est mis à disposition selon les termes de la licence Creative Commons Attribution - Pas d'Utilisation Commerciale - Pas de Modification 4.0 International. 


\title{
«On ne peut pas empêcher les concepts de voyager $»$ : un entretien avec Christine Delphy
}

"You Can't Stop Concepts from Travelling": an Interview with Christine Delphy

\author{
Marc Calvini-Lefebvre
}

1 Le concept de "patriarcat ", qui est au cœur de la conception de ce numéro spécial, doit beaucoup aux travaux de la sociologue Christine Delphy. Directrice de recherche émérite au CNRS, co-fondatrice de la revue Nouvelles Questions Féministes ${ }^{1}$ avec Simone de Beauvoir, elle est l'une des théoriciennes majeures du féminisme matérialiste, terme qu'elle inventa en $1975^{2}$. En 1970, elle publia une analyse pionnière du travail domestique gratuit fournit par les femmes au sein des ménages français, arguant qu'il s'agissait là de la base économique de la subordination des femmes aux hommes, sans distinction de classe sociale. Face à cette "oppression spécifique »" les femmes se devaient de donner la priorité à «la destruction totale du système de production et de reproduction patriarcal $»^{4}$, qu'elle désignait, dans une formule célèbre, comme «l'ennemi principal» du mouvement de libération des femmes. Nous avons souhaité la rencontrer pour évoquer avec elle la genèse de ce concept, certaines des critiques qui ont pu lui être adressée, son articulation avec d'autres notions et concepts qui ont été avancé pour faire sens des inégalités entre hommes et femmes, et la prolifération récente de son usage jusque dans les médias. Nous tenons à la remercier pour sa générosité, sa patience, et son souci constant de la clarté et de la précision du propos.

2 Marc Calvini-Lefebvre: Qu'entendez-vous par patriarcat?

3 Christine Delphy: Le mot patriarcat désigne un système, non pas dans sa manière de fonctionner, mais comme un état de fait, un état de domination masculine. On pourrait utiliser d'autres termes pour décrire ce système : sexiste, viriliste, excluant. On peut dire, par exemple, « le système politique en France est patriarcal ». Mais ce n'est pas la 
seule manière dont on peut le décrire, et ça n'épuise pas le mot de patriarcat. Un système peut être patriarcal et beaucoup d'autres choses.

$M C L$ : D'où vous est venu ce terme?

5 CD : Je ne suis pas historienne mais j'ai lu beaucoup de romans du $19^{\mathrm{e}}$ siècle. Chez des auteurs aussi différents que Hugo ou Marx, "patriarcal » désignait une espèce de société plus ou moins primitive et plus ou moins idéale où tout le monde dépendait de la bonne volonté d'un père de famille. Ce qui m'avait frappé c'est que les auteurs du $19^{\mathrm{e}}$ utilisaient beaucoup le terme de "vertu patriarcale » qui renvoyait à un mythe d'une société paysanne ou une famille heureuse vivait sous l'autorité d'un patriarche. Et l'image de ce patriarche c'était celle d'un gentil grand-père! C'est sans doute pour cela que, dans les années 60-70, même des collègues qui étaient très proches de moi, me disaient «mais patriarcat ça ne va pas pour désigner la domination des hommes parce que ça vient de pater, pater c'est le père, ce n'est pas le mari! » Ils pensaient au chef de famille. Or dans notre tradition, qui est la tradition de tous les pays occidentaux, et probablement de beaucoup d'autres, et bien le pater il est aussi le chef de la famille. Il n'est pas uniquement le géniteur des enfants.

$6 \quad$ MCL : Vous avez donc pris un concept courant du dix-neuvième siècle en l'investissant d'un sens nouveau, ce qui permet de suggérer une continuité dans le temps de ce système, malgré les profondes différences entre la France de Hugo et la France de Pompidou. Cette idée d'une continuité a pu être critiquée par certaines historiennes, comme Sheila Rowbotham ${ }^{5}$, qui ont reproché au concept de patriarcat de ne pas être en mesure de rendre compte du changement des sociétés dans le temps et à ce titre d'être anhistorique. Comment percevez-vous cette critique?

$7 \quad \mathrm{CD}$ : Je vous ferai une réponse en deux temps. Tout d'abord, quand je me suis intéressée très explicitement au $19^{e}$ siècle avec une collègue britannique, Diana Leonard ${ }^{6}$, nous avons étudié les données empiriques sur la production familiale/familière afin de dire quelque chose de l'organisation du système patriarcal de l'époque et non d'un système patriarcal abstrait, qui flotterait au-dessus de l'histoire. Loin d'être anhistorique, ce concept est, me semble-t-il, transhistorique. En effet, on ne peut soutenir qu'il est une création du $20^{\mathrm{e}}$, du $19^{\mathrm{e}}$ ou même du $18^{\mathrm{e}}$ siècle. Par exemple, certaines historiennes font dater la relégation politique des femmes à la Révolution Française, parce qu'elles trouvent cette philosophie chez Jean-Jacques Rousseau. Mais il ne la tenait pas de nulle part! Le sort des femmes avant la Révolution n'était pas non plus très enviable. C'est le problème de ce que j'appelle le présentéisme: on prétend chercher l'origine d'un phénomène, mais on n'ose remonter trop loin au prétexte qu'avant, on n'a pas de sources. Pourtant, il me semble que l'oppression des femmes est observable dans les sources qui nous restent du Haut Moyen-Age, du Bas Moyen-Age, chez les Romains, chez les Grecs, etc.... A chaque époque, ce que l'on retrouve, c'est un patriarcat. Le patriarcat, dans le sens large de domination, on ne peut pas dire que ce soit un phénomène si historiquement circonscrit que cela. Il est peut -être circonscrit mais nous ne savons pas encore où en sont les limites.

8 MCL: C'est une question que mes étudiant.e.s me posent souvent: " mais alors, ça remonte à quand l'inégalité femmes-hommes»?

9 CD : Oui, mais attention, je ne veux pas dire pour autant qu'il faille le faire remonter à la nuit des temps! Lorsque l'anthropologue Françoise Héritier, une vrai féministe, une femme sympathique, affirme que la domination masculine a commencé il y a 500000 ans, je trouve cela très problématique ${ }^{7}$. Comment le sait-elle? A partir de quelle base 
empirique ? Je constate pour ma part que, chaque fois que l'on découvre un nouveau tibia, cela change entièrement les dates de la préhistoire. Force est de constater que l'on ne connaît rien de ces sociétés : sont-elles déjà humaines? Sont-ce encore des singes? On n'en sait rien. Or, certains anthropologues font parfois comme si ce que ces personnes croyaient ce serait perpétué jusqu'à nos jours, sous la forme de tabous ancestraux autour de la menstruation ou de l'allaitement par exemple, et cela expliquerait l'organisation patriarcale. Mais d'où viennent-ils, ces tabous? Ils sortent de terre, comme des asperges? La culture n'est pas gratuite, elle n'apparaît pas comme ça. Elle fait partie de la société, elle ne lui est pas extérieure. Elle ne force pas la société. Les sociétés et leurs croyances sont toujours en phase. La culture c'est l'un des éléments de la société. On ne peut pas l'en distinguer, parce que ce sont les mêmes acteurs.

10 A ce premier point historique, j'en ajouterai un second, épistémologique. Souvent ce qui apparaît comme une querelle théorique ne l'est pas. En France, par exemple, j'ai pu constater, lorsque j'ai participé au petit groupe de l'Action Thématique Programmée du CNRS, un programme de recherche qui durait quatre ans, combien les historiennes présentes refusaient d'utiliser le mot " genre », lui préférant la version anglaise gender. Pourtant, chez les sociologues, le terme sous sa forme française était présent dans nos travaux depuis 15-20 ans ! C'est un mot dérivé du latin genus, un mot très ancien. On le retrouve dans "gente masculine ", "gente féminine ", et dans ce mot très répandu en France, «les gens»! Tout le monde faisait comme si c'était une importation de l'anglais. Ce n'est pas une importation de l'anglais. C'est l'utilisation d'un mot, comme l'ont fait les américaines et les anglaises d'ailleurs, pour signifier, pour ne pas le réduire au genre grammatical, c'est tout. Mais dire que cela venait de l'anglais, c'était une manière de le maintenir à distance, de montrer que l'on respectait les rapports de pouvoir dans le champ. Je ne sais pas si c'est le cas pour Sheila Rowbotham, mais en France, en tout cas, il me semble qu'il a été plus difficile pour les historiennes de se faire entendre dans leur champ que les sociologues.

11 MCL: Vous avez aussi été pionnière dans la théorisation du genre en Frances. Comment l'articulez-vous dans vos travaux avec le concept de patriarcat?

CD : Les deux vont ensemble, mais ne sont pas identiques. On peut parler du patriarcat de la France, pas du genre de la France. Constater la présence d'un système patriarcal relève de la prise d'un instantané, d'une photo. Mais s'intéresser au genre c'est entrer dans l'ordre de l'analyse du système de partition qui créé deux catégories de personnes et les hiérarchise.

MCL: Dans l'ennemi principal vous distinguiez le système patriarcal du système capitaliste, arguant de leur autonomie réciproque. Dans ce numéro spécial, de nombreuses contributions s'interrogent sur les conséquences du tournant néolibéral des années 1980 initié par Margaret Thatcher, sur le statut des femmes au Royaume-Uni. Quel regard portez-vous sur cette question?

CD : Capitalisme et patriarcat sont deux systèmes indépendants mais qui s'appuient mutuellement. Si le genre des gens était indifférent pour le capitalisme, il ne prendrait pas le parti du patriarcat, les choses ne seraient pas semblables. Néolibéralisme est un nouveau mot. Le risque de ces nouveaux mots c'est de donner l'impression que l'on est dans une autre ère. Mais nous ne le sommes pas. Lorsque j'étais à la Fondation Copernic, j'ai eu à écrire une introduction pour un ouvrage qui s'interrogeait sur le sort de tout le monde, pas que des femmes, dans ce qu'il appelait «l'ère néolibérale ». J'ai demandé à mon co-président pourquoi les contributrices et contributeurs de cette 
ouvrage ne s'attaquaient pas plutôt au capitalisme. Et il m'avait répondu que c'était parce qu'ils et elles considéraient que ce n'était pas la même chose. Pour moi, c'est la même chose. Ce n'est qu'un développement du capitalisme. C'est voir les choses par le petit bout de la lorgnette.

Ceci étant dit, la situation des femmes se détériore évidemment depuis le tournant Thatchérien/Reaganien, y compris en France. Le but est toujours de limiter l'action de l'Etat pour développer l'économie. C'est un leitmotiv que l'on connaît depuis 40 ans. Cette politique agit d'une façon spécifique sur les femmes parce qu'elles sont spécifiques, parce qu'elles partent avec un fardeau beaucoup plus lourd, ça leur pèse plus qu'aux autres. Mais tout le monde est affecté.

MCL: Pour faire sens et combattre les inégalités que le moment néolibéral génère ou aiguise, genre, comme patriarcat, sont des concepts qui, pour le meilleur et pour le pire, sont passés dans le discours médiatique, voire même dans la pop culture. Craignez-vous que ces concepts perdent de leur tranchant analytique en se diffusant ainsi?

$\mathrm{CD}$ : On ne peut pas empêcher les concepts de voyager! Ils vont voyager, et après il y aura des débats et des débats qui n'aboutiront pas à un emploi unanime. En fait, ce qu'il faut faire c'est dire "voilà ce que moi j'entends par là ». On ne peut pas faire autrement!

MCL: Voyageons alors avec ce concept pour tenter d'éclairer notre actualité. Lors de l'élection présidentielle américaine récente, les femmes qui se sont déplacées ont, très majoritairement, votées pour Hillary Clinton. Cependant, lorsque l'on a regardé plus finement le vote par catégorie ethnique, on voit que les femmes blanches font exception: elles ont voté à $53 \%$ pour Donald Trump. La sociologue Deniz Kandyioti a proposé la notion de " troc patriarcal " " pour expliquer le soutien de certaines femmes au système patriarcal : parce qu'elles en tirent certains avantages matériels et de statut, elles lui apporteraient leur soutien malgré leur position subordonnée par rapport aux hommes de leur catégorie sociale. Cette notion vous parait-elle éclairante pour ce cas?

$\mathrm{CD}$ : Je dirai d'abord que les femmes blanches ne sont pas plus responsables de l'élection de Trump que les hommes blancs! Ensuite, cette notion de troc patriarcal, très intéressante, rejoint me semble-t-il les analyses d'Andréa Dworkin dans son ouvrage Les Femmes de Droite ${ }^{10}$ : elle disait des femmes de droite qu'elles soutenaient le vote de leurs hommes de droite pour ne pas "rock the boat ». Leur intérêt était de passer dans la catégorie la moins opprimée de femmes, c'est-à-dire de femmes épousant un homme relativement aisé.

Ainsi, dans tous les groupes dominés, il y a des personnes qui peuvent penser marginalement bénéficier de régimes qui a priori sembleraient les opprimer, parce qu'elles ne se voient pas comme faisant partie d'une catégorie (les femmes) plus grande que leur petite catégorie (les femmes blanches) mais plutôt comme étant en compétition avec tous les membres de cette catégorie. C'est exactement le même problème qui se pose à propos de la race, et on voit bien les résultats dans notre pays : on ne dit pas « ce sont les patrons qui nous exploitent », on dit : « les patrons emploient des Arabes et des Noirs qui nous prennent notre travail». Conclusion: il faut se débarrasser de cette concurrence pour avoir une part plus grande de ce petit quart de gâteau qui est ce qui nous est échu. Mais l'on ne remet pas en cause la taille de la part. Ainsi, ce que je mettrai en avant c'est plutôt cette compétition interne dans une classe 
qui ne se connaît pas comme classe. Ou plutôt, qui se connaît comme classe en soi mais a renoncé, n'a même jamais envisagé, de lutter pour sa libération.

MCL : La misogynie, l'anti-féminisme étaient au coeur de la campagne de Donald Trump et on les retrouve aussi dans des mouvements réactionnaires un peu partout en Europe. Peut-on parler, d'après-vous, d'une nouvelle période de "backlash ", après celle identifiée par Susan Faludi dans les années 1980-1990 aux Etats-Unis ${ }^{11}$ ?

CD : Vous savez, le backlash, en France, le mouvement féministe l'a vécu dès son apparition. Mais que pouvait-on attendre d'autre? Le backlash est inévitable et permanent. Dès qu'une catégorie de gens essaient de lever le joug qui pèse sur leurs cous il ne faut pas penser que les autres vont accepter de bonne humeur. Lorsque Susan Faludi a écrit son ouvrage, elle découvrait peut-être ce phénomène, alors en a donné toutes les preuves. Mais ces preuves ne manquent pas, elles sortent tous les jours! Et pas seulement contre les femmes, contre toutes les personnes stigmatisées qui tentent de s'organiser. Chaque fois qu'il y a un mouvement de libération, de protestation, des catégories dominées il y a une réaction des catégories dominantes. On ne peut pas s'attendre à autre chose. Malheureusement les femmes, elles, s'attendent trop souvent à autre chose parce que, contrairement à d'autres groupes dominés, elles sont très nombreuses à vivre et à avoir des relations affectives très fortes, avec les membres du groupe dominant.

Marc Calvini-Lefebvre est Maître de conférences en civilisation britannique du XIXe siècle à Aix-Marseille Université. Chercheur au Laboratoire d'Etudes et de Recherche sur le Monde Anglophone (LERMA, EA 853). Ses travaux portent sur l'histoire intellectuelle du féminisme britannique.

\section{BIBLIOGRAPHIE}

ALEXANDER Sally \& TAYLOR Barbara, «In defence of "patriarchy" ", The New Statesman, February 1, 1980, p. 161.

DELPHY Christine, L'ennemi principal. Tome 1 : économie politique du patriarcat, Paris, Edition Syllepse, $3^{\mathrm{e}}$ édition, 2013.

- L’ennemi principal. Tome 2 : penser le genre, Paris, Edition Syllepse, $3^{\mathrm{e}}$ édition, 2013.

- \& LEONARD Diana, Familiar Exploitation. A new analysis of marriage in contemporary western societies, Cambridge (U.K.), Polity Press, 1992.

DWORKIN Andrea, Right wing women, New York, Perigee Books, 1983.

HÉRITIER Françoise, Masculin/Féminin, tome 1 : la pensée de la différence, Paris, Odile Jacob, 2007.

- Masculin/Féminin, tome 2 : dissoudre la hiérarchie, Paris, Odile Jacob, 2012.

KANDIYOTI Deniz, « Bargaining with patriarchy », Gender and Society, Vol. 2, No. 3, Special Issue to Honor Jessie Bernard, Sep., 1988, p. 274-290. 
FALUDI Susan, Backlash: the undeclared war against American women, London, Chatto \& Windus, 1992.

ROWвотнАм Sheila, " The trouble with "patriarchy" ", The New Statesman, 21/28 December 1979.

\section{NOTES}

1. https://www.cairn.info/revue-nouvelles-questions-feministes.htm (dernière consultation: 27/11/2017)

2. DELPHY Christine, « Pour un féminisme matérialiste », in DELPHY Christine, L'ennemi principal. Tome 1 : économie politique du patriarcat, Paris, Edition Syllepse, 2013, $3^{\mathrm{e}}$ édition, p. 243-253. Cet article est paru pour la première fois dans L'Arc, en avril 1975.

3. DELPHY Christine, «Le patriarcat : une oppression spécifique », in DELPHY Christine, L'ennemi principal. Tome 2: penser le genre, Paris, Edition Syllepse, 2013, $3^{\mathrm{e}}$ édition, p. 51-83. Cet entretien avec Louis Astre et Liliane Kandel, avait été publié à l'origine en 1988 dans Le féminisme et ses enjeux, FEN, Edilig.

4. DELPHY Christine, «L'ennemi principal », in DELPHY Christine, L'ennemi principal. Tome 1: économie politique du patriarcat, Paris, Edition Syllepse, 2013, 3e édition, p. 52. Cet article est apparu pour la première fois en 1970 dans un numéro spécial de la revue Partisans intitulé : « Libération des femmes. Année zéro ».

5. ROWBOтнAм Sheila, "The trouble with "patriarchy" ", The New Statesman, 21/28 December 1979. Pour une réponse contemporaine, voir Sally Alexander \& Barbara Taylor, «In defence of "patriarchy" ", The New Statesman, February 1, 1980, p. 161.

6. DELPHY Christine \& LEONARD Leonard, Familiar exploitation. A new analysis of marriage in contemporary western societies, Cambridge (U.K.), Polity Press, 1992.

7. Voir HÉRITIER Françoise, Masculin/Féminin, tome 1: la pensée de la différence, Paris, Odile Jacob, 2007 et Masculin/Féminin, tome 2 : dissoudre la hiérarchie, Paris, Odile Jacob, 2012.

8. DELPHY Christine, « Penser le genre : problèmes et résistances ", in DELPHY Christine, L'ennemi principal. Tome 2: penser le genre, Paris, Edition Syllepse, 2013, $3^{\mathrm{e}}$ édition, p. 223-238. Cet article était paru sous le titre «Penser le genre : quels problèmes? » dans HURTIG M.C. et al. (eds.), Sexe et genre, Paris, Presses du CNRS, 1991.

9. KANDIYOTI Deniz, « Bargaining with patriarchy », Gender and Society, Vol. 2, No. 3, Special Issue to Honor Jessie Bernard (Sep., 1988), p. 274-290.

10. DWORKIN Andrea, Right wing women, New York, Perigee Books, 1983.

11. FALUDI Susan, Backlash: the undeclared war against American women, London, Chatto \& Windus, 1992.

\section{RÉSUMÉS}

Le concept de "patriarcat", qui est au cœur de la conception de ce numéro spécial, doit beaucoup aux travaux de la sociologue Christine Delphy. En 1970, elle publia une analyse pionnière du travail domestique gratuit fournit par les femmes au sein des ménages français, arguant qu'il s'agissait là de la base économique de la subordination des femmes aux hommes, 
sans distinction de classe sociale. Face à cette "oppression spécifique ", les femmes se devaient de donner la priorité à «la destruction totale du système de production et de reproduction patriarcal ", qu'elle désignait, dans une formule célèbre, comme «l'ennemi principal » du mouvement de libération des femmes. Nous avons souhaité la rencontrer pour évoquer avec elle la genèse de ce concept, certaines des critiques qui ont pu lui être adressée, son articulation avec d'autres notions et concepts qui ont été avancé pour faire sens des inégalités entre hommes et femmes, et la prolifération récente de son usage jusque dans les médias.

The concept of patriarchy which is at the heart of this special issue, owes a great deal to the work of French sociologist Christine Delphy. In 1970, she published a pioneering analysis of the free labour that women did in the home, arguing that it was the economic foundation of their subordination to men, whatever their social class. In the face of this "sex-specific oppression", women must prioritise, she argued, the "total destruction of the system of patriarchal production and reproduction, a system which she famously called the "principal enemy" of the women's liberation movement. We wished to meet her to ask her about the genesis of this concept, its articulation with other notions and concepts that have been put forward to make sense of inequalities between women and men, and the recent proliferation of its usage in pop culture and the mainstream media.

\section{INDEX}

Mots-clés : patriarcat, genre, backlash, capitalisme, néolibéralisme

Keywords : patriarchy, gender, backlash, capitalism, neoliberalism

\section{AUTEUR}

\section{MARC CALVINI-LEFEBVRE}

Laboratoire d'Etudes et de Recherche sur le Monde Anglophone (LERMA), EA 853, 13625, AixMarseille Université. 